\title{
Analysis of the impact of antidepressants and other medications on COVID-19 infection risk in a chronic psychiatric in-patient cohort
}

\author{
Catherine L. Clelland, Krista Ramiah, Louisa Steinberg and James D. Clelland
}

\section{Background}

During the first wave of the coronavirus disease 2019 (COVID-19) pandemic, patients with confirmed cases in New York State accounted for roughly $25 \%$ of total US cases, with psychiatric hospital in-patients at particularly high risk for COVID-19 infection.

\begin{abstract}
Aims
The beneficial effects of mental health medications, such as selective serotonin reuptake inhibitors (SSRIS), on the severity of COVID-19 disease outcomes have been documented. Protective effects against infection have also been suggested for these medications. We therefore tested the hypothesis that medication use modifies the risk of COVID-19 infection in a long-stay, chronic in-patient psychiatry setting, where the potential for exposure was likely uniform across the facility, and where these medications were routinely prescribed.
\end{abstract}

\section{Method}

This was a retrospective cohort study of an adult psychiatric facility operated by the New York State Office of Mental Health. Current medication information and COVID-19 status was collected from electronic medical records for 165 people who were in-patients during the period January to July 2020, and logistic regression was employed to model the main effects of medication use on COVID-19 infection.

\section{Results}

A significant protective association was observed between antidepressant use and COVID-19 infection (odds ratio $(\mathrm{OR})=0.33$, $95 \% \mathrm{Cl} 0.15-0.70$, adjusted $P<0.05$ ). Analysis of individual antidepressant classes showed that SSRI, serotonin-norepinephrine reuptake inhibitor and the serotonin-2 antagonist reuptake inhibitor classes of antidepressants, drove this protective effect. Exploratory analyses of individual antidepressants demonstrated an association between lower risk of infection and fluoxetine use $(P=0.023)$, as well as trazodone use $(P=0.001)$.

\section{Conclusions}

The novel finding of reduced COVID-19 infection risk for psychiatric in-patients taking antidepressants, suggests that antidepressants may be an important weapon in the continued fight against COVID-19 disease. This finding may become particularly salient for in-patient settings if vaccine-resistant strains of the virus appear.

\section{Keywords}

Antidepressants; fluoxetine; trazodone; psychiatric illness; COVID-19.

\section{Copyright and usage}

(c) The Author(s), 2021. Published by Cambridge University Press on behalf of the Royal College of Psychiatrists. This is an Open Access article, distributed under the terms of the creative Commons Attribution-NonCommercial-NoDerivatives licence (https://creativecommons.org/licenses/by-nc-nd/4.0/), which permits non-commercial re-use, distribution, and reproduction in any medium, provided the original work is unaltered and is properly cited. The written permission of Cambridge University Press must be obtained for commercial re-use or in order to create a derivative work.

\section{Background}

During the first wave of the coronavirus disease 2019 (COVID-19) pandemic, caused by the severe acute respiratory syndrome coronavirus 2 (SARS-CoV-2), patients with confirmed cases in New York State accounted for roughly $25 \%$ of total COVID-19 cases in the USA. Psychiatric hospital in-patients were at particularly high risk for COVID-19 infection, with rates approaching those found in nursing homes. ${ }^{1}$ Patient populations with serious mental illness may be particularly vulnerable to severe infection because close to half of these patients have one or more comorbidities, ${ }^{2}$ and in fact those with schizophrenia spectrum disorder have an increased risk of COVID-19 mortality. ${ }^{3}$

Since early 2020 when the pandemic began there have been several observational studies examining the potential benefits of mental health medications, such as antipsychotics and antidepressants, on the course and severity of COVID-19 disease outcomes. Regarding antipsychotics, although theorised to have beneficial effects, ${ }^{4}$ observational studies of antipsychotic treatment have to date shown no significant benefit on severe outcomes of COVID19 disease. ${ }^{5,6}$ On the other hand, two large observational studies conducted in Europe (France and Spain), both demonstrated the significant, and positive association between antidepressant use and a reduced risk of intubation or death in patients admitted to hospital with COVID-19. ${ }^{7,8}$ This data is strongly supported by the encouraging findings from clinical trials of the selective serotonin reuptake inhibitor (SSRI) fluvoxamine in the USA, in which treated patients exhibited a significantly lower likelihood of clinical deterioration, ${ }^{9}$ or showed improved respiratory rates and significantly lower incidence of subsequent admission to hospital approximately 2 weeks after the onset of treatment. ${ }^{10}$

It has also been postulated that some medications may alter the risk of COVID-19 infection following exposure by obstructing SARS-CoV-2 host cell entry, which occurs via the virus binding to the membrane-bound angiotensin-converting enzyme 2 (ACE2) in the nasal passages and lungs. For example, supplementation with vitamin $\mathrm{D}$ has been hypothesised to decrease the risk of infection by normalising ACE2 function following exposure, ${ }^{11}$ and supporting this hypothesis, two large general population cohort studies, one in the UK ${ }^{12}$ and the other in Spain, ${ }^{13}$ documented a small, but protective effect of vitamin D supplementation on COVID-19 positivity. Of particular interest, in light of the positive effects of antidepressants on the course of COVID-19 disease outcomes, ${ }^{7,8}$ data from a recent in vitro study showed that some antidepressants may also prevent SARS-CoV-2 cell entry via inhibition of the acid sphingomyelinase (ASM)/ceramide system, ${ }^{14}$ which is likely required to facilitate ACE2 binding of the SARS-CoV-2 virus. ${ }^{15}$ Furthermore, there is a substantial body of work documenting the 
many antiviral properties of antidepressants, in particular SSRIs and it has been theorised that antidepressants may be effective against SARS-CoV-2 infection. ${ }^{16,17}$

\section{Aims}

Intriguingly, recent population cohort studies investigating medication effects on COVID-19 risk ${ }^{12,13,18}$ may be confounded by actual rates of viral exposure in the broad geographical communities studied. We propose that risk of infection could also be examined in an in-patient population setting, where the potential for exposure is likely uniform across the facility, and where these mental health medications/supplements are routinely prescribed. In the present study we tested the hypothesis that medication use, such as antidepressants or vitamin D supplementation, modifies the risk of COVID-19 infection in a long-stay, chronic in-patient psychiatry setting.

\section{Method}

We conducted a retrospective cohort study of the in-patient population at The Rockland Psychiatric Center (RPC), a large psychiatric facility for adults operated by the New York State Office of Mental Health $(\mathrm{OMH})$. The authors assert that all procedures contributing to this work comply with the ethical standards of the relevant national and institutional committees on human experimentation and with the Helsinki Declaration of 1975, as revised in 2008. All procedures involving human patients were approved by the RPC institutional review board, using a waiver of authorisation for informed consent. The study followed the STROBE reporting guidelines for cohort studies.

During the period between June 2 to 31 July 2020, we collected patient data from the $\mathrm{OMH}$ online medical records system, across seven wards in entirety (approximately $50 \%$ of the RPC in-patient population). Demographic and clinical details were recorded, plus all current medication use (including PRN medications) within the first wave of the pandemic in New York State. COVID-19 infection was determined by a positive polymerase chain reaction diagnostic test or the presence of antibodies following an enzyme-linked immunosorbent assay. Testing was performed at RPC for all patients from the period 24 March 2020 to 31 July 2021. Those who had refused to be tested $(n=5)$ were excluded from our analysis.

Fisher's exact or Student's $t$-tests were employed to compare demographic and clinical characteristics of the RPC sample, by COVID-19 infection status. Logistic regression was then employed to model the main effects of medication on the primary outcome of COVID infection (yes/no). Common medications were tested in the following groups; typical neuroleptic use (yes/no), atypical neuroleptic use (yes/no) and chlorpromazine-equivalent (CPZE) (yes/ no) daily dose, mood stabiliser (yes/no), antidepressant (yes/no), benzodiazepine (yes/no), anticholinergic (yes/no), antilipidemic (yes/no), antihypertensive (yes/no), antibiotics (yes/no), antiviral (yes/no), steroids (yes/no) and supplement (yes/no). To control the false discovery rate (FDR, $<5 \%), P$-values were subjected to Benjamini-Hochberg adjustment. Following the statistical analysis strategy employed by Nemani et $\mathrm{al}^{3}$, when significant medication main effects were observed (Benjamini-Hochberg $P<0.05$ ), patient-level characteristics considered related to COVID-19 infection were employed as covariates in demographic-adjusted models (age, gender, ethnicity, psychiatric diagnosis, ward and body mass index (BMI)), followed by fully adjusted models with the additional clinical variables of diabetes, hypertension, respiratory illness and heart disease. Current smoking status was not available for most in-patients from online records and was thus not investigated. As a sensitivity analysis, a stepwise selection procedure (criteria set to $P<0.05$ ) was employed, starting with all variables from the fully adjusted model. In exploratory analyses Fisher's exact tests were employed to test for the association between classes of and individual antidepressant and antipsychotic medication use (when the total sample treated was $\geq n=5$ ) and COVID-19. All analyses were conducted in Stata v14.2 (Stata, College Station TX).

\section{Results}

A total of 165 RPC in-patients were included (Table 1), of whom 91 (55\%) were positive for COVID-19. Individuals with COVID-19 did not differ by gender, BMI or the presence of hypertension, respiratory illness or diabetes. There was a trend towards significance for COVID19 positively to be associated with schizophrenia spectrum disorders (schizophrenia or schizoaffective disorder) when compared with

Table 1 Demographic and clinical characteristics of the RPC sample

$(n=165)$

\begin{tabular}{|c|c|c|c|}
\hline Characteristic & $\begin{array}{l}\text { COVID+ } \\
(n=91)\end{array}$ & $\begin{array}{l}\text { COVID- } \\
(n=74)\end{array}$ & $P^{\mathrm{a}}$ \\
\hline Gender, n (row \%) & & & 0.327 \\
\hline Female & $8(12)$ & $11(58)$ & \\
\hline Male & $83(57)$ & $63(43)$ & \\
\hline Ethnicity, $n$ (row \%) & & & 0.671 \\
\hline African American & $43(41)$ & $30(59)$ & \\
\hline White & $39(52)$ & $36(48)$ & \\
\hline Other & $8(47)$ & $9(53)$ & \\
\hline Age, years, $n$ (row \%) & & & 0.095 \\
\hline $18-44$ & $29(45)$ & $36(55)$ & \\
\hline $45-54$ & $22(69)$ & $10(31)$ & \\
\hline $55-64$ & $26(55)$ & $21(45)$ & \\
\hline $65+$ & $14(67)$ & $7(33)$ & \\
\hline BMI, n (row \%) & & & 0.534 \\
\hline Normal $(\mathrm{BMI} \leq 25)$ & $23(59)$ & $16(41)$ & \\
\hline Overweight (BMI 26-30) & $40(58)$ & $29(42)$ & \\
\hline Obese (BMI 31+) & $28(49)$ & $29(51)$ & \\
\hline Psychiatric diagnosis, ${ }^{b} n$ (row \%) & & & 0.062 \\
\hline Schizophrenia spectrum disorder & $84(58)$ & $61(42)$ & \\
\hline Mood disorder & $5(31)$ & $11(69)$ & \\
\hline Hypertension, n (row \%) & & & 0.160 \\
\hline Yes & $29(64)$ & $16(36)$ & \\
\hline No & $61(51)$ & $58(49)$ & \\
\hline Respiratory illness, n (row \%) & & & 0.756 \\
\hline Yes & $7(64)$ & $4(36)$ & \\
\hline No & $84(55)$ & $70(45)$ & \\
\hline Diabetes (type 2), n (row \%) & & & 0.185 \\
\hline Yes & $10(42)$ & $14(48)$ & \\
\hline No & $81(57)$ & $60(43)$ & \\
\hline Heart disease, $n$ (row \%) & & & 0.062 \\
\hline Yes & $5(31)$ & $11(69)$ & \\
\hline No & $86(58)$ & $63(42)$ & \\
\hline \multicolumn{4}{|l|}{ Medications } \\
\hline CPZE, mean (s.d.) & $1729.4(5365.8)$ & $737.8(629.7)$ & 0.116 \\
\hline Typical neuroleptic, yes: n (row \%) & $54(61)$ & 34 (39) & 0.084 \\
\hline Atypical neuroleptic, yes: $n$ (row \%) & $85(54)$ & $71(46)$ & 0.733 \\
\hline Mood stabiliser, yes: $n$ (row \%) & $52(55)$ & $42(45)$ & 1.000 \\
\hline Benzodiazepine, yes: $n$ (row \%) & $43(65)$ & $23(35)$ & 0.039 \\
\hline Antidepressant, yes: n (row \%) & $13(34)$ & $25(66)$ & 0.002 \\
\hline Anticholinergic, yes: n (row \%) & $47(59)$ & $32(41)$ & 0.347 \\
\hline Antilipidemic, yes: $n$ (row \%) & $21(50)$ & $21(50)$ & 0.475 \\
\hline Antihypertensive, yes: $n$ (row \%) & $34(60)$ & $23(40)$ & 0.596 \\
\hline Antibiotic, yes: $n$ (row \%) & $1(50)$ & $1(50)$ & 1.000 \\
\hline Antiviral, yes: $n$ (row \%) & $3(75)$ & $1(25)$ & 0.628 \\
\hline Steroid, yes: $n$ (row \%) & $12(57)$ & $9(43)$ & 1.000 \\
\hline Supplement, ${ }^{c}$ yes: $n$ (row \%) & $54(63)$ & $32(37)$ & 0.043 \\
\hline
\end{tabular}

$\mathrm{BMI}$, body mass index; CPZE, chlorpromazine-equivalent daily dose. Significant differences bolded.

a. Fisher's exact or Student's $t$-test.

b. $n=161$ (four participants with unspecified psychiatric diagnoses were excluded)

c. Vitamins B (B complex, B12, folic acid), C, D, multivitamin, fish oil, Calcium carbonate, magnesium, probiotic 
mood disorders (bipolar disorder or major depressive disorder) $(P=$ 0.062), supporting data from a recent study. ${ }^{3}$ There was no difference in COVID-19 infection status when patient diagnoses were further classified into manic and depressive types (see Supplementary Tables 1 and 2 available at https://doi.org/10.1192/bjo.2021.1053).

We observed a significant association of CPZE dose with increasing COVID-19 risk (Table 2, odds ratio $(\mathrm{OR})=1.0007$, 95\% CI 1.0002-1.0013, Benjamini-Hochberg adjusted $P<0.05$ ), which was retained after adjustment for demographic and clinical variables. Interpreting CPZE quartile data from the adjusted model, the probability of being COVID-19 positive increased from 0.41 at the 25th percentile, to 0.44 at the 50th, 0.52 at the 75th, to 0.63 at the 90th percentile of the CPZE daily dose data. The use of benzodiazepines and supplements were also associated with COVID-19 positivity but did not remain significant following Benjamini-Hochberg adjustment.

There was a significant association with antidepressant use (OR $=0.33,95 \%$ CI $0.15-0.70$, Benjamini-Hochberg adjusted $P<0.05$ ); those treated with antidepressants had significantly reduced odds of COVID-19 infection, even after full adjustment for all demographic and clinical variables (fully adjusted $\mathrm{OR}=0.28$, Table 2 ). Results from sensitivity analyses using a stepwise selection of model variables yielded similar results. Analysis of individual antidepressant classes showed that patients treated with serotonin reuptake inhibitors (SSRIs and serotonin-norepinephrine reuptake inhibitors (SNRIs)) or serotonin-2 antagonist reuptake inhibitors (SARI), both had a significantly decreased likelihood of COVID19 infection (Table 2: unadjusted OR $=0.32,95 \%$ CI $0.12-0.78$ and $\mathrm{OR}=0.06,95 \%$ CI 0.008-0.51, respectively). Exploratory analyses of individual antidepressant use demonstrated a significant association between lower risk of infection and the SSRI antidepressant fluoxetine ( $P=0.023)$, as well as the SARI antidepressant trazodone $(P=0.001)$, see Supplementary Table 3.

\section{Discussion}

\section{Main findings}

In this observational study of a long-stay hospital, psychiatric inpatient cohort, we found that patients who received antidepressant medication had a $72 \%$ lower odds of testing positive for COVID-
19, compared with those not treated with antidepressants. The serotonin reuptake inhibitor (SSRI and SNRI) and the SARI classes of antidepressants appeared to drive the protective effect. Our finding augments recent studies documenting the beneficial effect of antidepressants (including both SSRIs and non-SSRIs) on reducing the risk of intubation or death in patients admitted to hospital with COVID$19,{ }^{7,8,19}$ and taken together provides support for a randomised controlled trial to test the use of these antidepressants in the management of COVID-19 risk in psychiatric in-patient settings.

\section{Interpretation of our findings}

There are a number of plausible pathophysiological mechanisms that could explain the protective effects of antidepressant medication against COVID-19 infection. First, antidepressant use may directly impede viral host cell entry via inhibition of the ASM/ceramide system ( ${ }^{20,21}$ and references therein). Specifically, the ASM enzyme, present in lysosomes and the cell membrane, cleaves ceramide from sphingomyelin, resulting in the formation of ceramide-enriched membrane domains in the outer cell membrane. Preclinical studies have suggested that SARS-CoV-2 infection requires activation of the ASM/ceramide system, ${ }^{14}$ with viral entry into host cells facilitated by the clustering of ACE2 into these ceramide-enriched membrane domains. ${ }^{15}$ Of relevance to our study finding, several antidepressants including the SSRI fluoxetine functionally inhibit ASM activity, ${ }^{14}$ and pharmacological in vitro studies have shown that treatment with a number of both SSRI, tricyclic and tetracyclic antidepressants directly block uptake of SARS-CoV-2 by epithelial cells, ${ }^{14}$ and with regards to fluoxetine, ${ }^{22,23}$ also dramatically reduced viral titers. ${ }^{24}$

Studies of both cell culture systems and in vivo models have also highlighted the antiviral activity of particular antidepressants. For example, fluoxetine is a potent inhibitor of enterovirus replication, ${ }^{25,26}$ the SSRI sertraline can inhibit Ebola virus cell entry both in vitro and in vivo, ${ }^{27}$ the SSRI citalopram inhibits HIV cell entry and replication, ${ }^{28}$ and citalopram and sertraline may reduce HIV replication in patient cerebrospinal fluid. ${ }^{29}$ Antidepressants can also act as anti-inflammatory agents, reducing levels of proinflammatory cytokines. ${ }^{17}$ For example, binding of fluoxetine to the sigma-1 receptor in the endoplasmic reticulum was shown to decrease cytokine activity and enhance survival in preclinical models of sepsis and inflammation, ${ }^{30}$ and human studies have supported the concept of a general decrease

\begin{tabular}{|c|c|c|c|c|c|c|}
\hline \multirow[t]{2}{*}{ Medication } & \multicolumn{2}{|c|}{ Unadjusted model } & \multicolumn{2}{|l|}{ Adjusted model ${ }^{\mathrm{a}}$} & \multicolumn{2}{|c|}{ Fully adjusted model ${ }^{b}$} \\
\hline & OR (95\% Cl) & $P$ & OR $(95 \% \mathrm{Cl})$ & $P$ & OR $(95 \% \mathrm{Cl})$ & $P$ \\
\hline CPZE & $1.0007(1.0002-1.0013)$ & $0.004^{\mathrm{C}}$ & $1.0007(1.0002-1.0014)$ & 0.044 & 1.0007 (1.0001-1.0015) & $0.046^{d}$ \\
\hline Typical antipsychotic & $1.765(0.947-3.287)$ & 0.073 & & & & \\
\hline Mood stabiliser & $1.016(0.547-1.888)$ & 0.960 & & & & \\
\hline Benzodiazepine & $1.986(1.046-3.773)$ & 0.036 & & & & \\
\hline Anticholinergic & $1.402(0.757-2.598)$ & 0.283 & & & & \\
\hline Antilipidemic & $0.757(0.375-1.528)$ & 0.438 & & & & \\
\hline Antihypertensive & $1.322(0.690-2.534)$ & 0.399 & & & & \\
\hline Antibiotic & $0.811(0.05-13.19)$ & 0.883 & & & & \\
\hline Antiviral & $2.489(0.253-24.436)$ & 0.434 & & & & \\
\hline Steroid & $1.097(0.435-2.765)$ & 0.844 & & & & \\
\hline Supplement & $1.916(1.029-3.567)$ & 0.040 & & & & \\
\hline Antidepressant & $0.327(0.153-0.698)$ & $0.004^{\mathrm{C}}$ & $0.357(0.132-0.966)$ & 0.042 & $0.280(0.094-0.837)$ & $0.023^{\mathrm{e}}$ \\
\hline SSRI/SNRI & $0.302(0.120-0.780)$ & 0.013 & & & & \\
\hline SARI & $0.064(0.008-0.505)$ & 0.009 & & & & \\
\hline \multicolumn{7}{|c|}{$\begin{array}{l}\text { Significant models are in bold. CPZE, chlorpromazine-equivalent daily dose; SSRI, selective serotonin reuptake inhibitor; SNRI, serotonin-norepinephrine reuptake inhibitor; SARI, serotonin-2 } \\
\text { antagonist reuptake inhibitor. } \\
\text { a. Adjusted for age (categorical: } 18-44 \text { years (reference group), } 45-54,55-64,65+) \text {; gender; ethnicity (categorical: African American (reference group), White, Other); psychiatric diagnosis; } \\
\text { ward (categorical: } 7 \text { levels), BMI (ordinal: normal, overweight, obese). } \\
\text { b. Adjusted as for footnote a, plus for the presence of diabetes, hypertension, respiratory illness or heart disease. } \\
\text { c. Benjamini-Hochberg adjusted } P<0.05 \\
\text { d. Stepwise regression model OR }=1.0007(1.000004-1.0014), P=0.049 \text {. } \\
\text { e. Stepwise regression model OR }=0.292(0.102-0.833), P=0.021 \text {. }\end{array}$} \\
\hline
\end{tabular}


of interleukin (IL)- $1 \beta$ and IL-6 in serum from patients taking antidepressants. ${ }^{31}$ Taken together these studies provide underlying mechanisms for the positive effect of antidepressant use on COVID-19 risk following potential exposure.

Typical antipsychotics have also been theorised to treat COVID19 symptoms, ${ }^{4}$ but so far observational studies of antipsychotic treatment (chlorpromazine ${ }^{5}$ or haloperidol ${ }^{6}$ ) have not demonstrated a beneficial effect of antipsychotic treatment on COVID-19 disease outcomes and overall mortality. In fact, one large cohort study of adult in-patients with a diagnosis of COVID-19 reported that antipsychotic treatment during COVID admission was significantly associated with a higher mortality rate. ${ }^{8}$ In our study, we observed a small, but significant association of increasing CPZE daily dose with increased COVID-19 infection, suggesting that use of some antipsychotics may also increase the risk of infection.

Consistent with a prior patient cohort study, ${ }^{18}$ we did not find an association between use of antihypertensive medication and COVID19. Conversely, our findings do not support previous population cohort studies documenting a protective effect of vitamin D supplementation on COVID-19 risk. ${ }^{12,13}$ Intriguingly, Meltzer et al, recently reported data collected from a large urban academic medical centre, showing an increased risk for testing positive for COVID-19 in patients with a likely vitamin D deficiency, compared with those with sufficient levels. ${ }^{32}$ We and others have reported that vitamin D deficiency is frequent in psychiatric in-patient populations. ${ }^{33}$ Thus, an explanation for our finding of significantly more patients treated with supplements (including vitamin D) who were COVID-19 positive, may be because of underlying deficiencies that negatively have an impact on COVID-19 risk in this long-stay psychiatric facility.

\section{Limitations}

The main limitation of this study was the small size of the in-patient sample investigated. Additionally, severity outcomes following infection were not analysed, primarily because most patients with severe illness were transferred to local hospitals with limited ongoing status reporting in $\mathrm{OMH}$ records. Additionally, one of the main hypotheses of this study was that the potential for exposure to COVID-19 was uniform across the RPC facility. Although group programmes at RPC were discontinued in late March 2020, prior to this period we were not able to collect information on daily activities and interactions that could influence risk, which is a limitation of the retrospective chart review protocol. Of relevance to this point, we did not find a significant difference in COVID-19 infection status between patients with a current depressive episode, current mania or psychosis, suggesting that in this study there was not a significant impact on COVID-19 infection status by individual psychopathology.

\section{Implications}

A follow-on large cohort study that also evaluates characteristics of COVID-19 infection would be beneficial to confirm these initial but interesting findings, with the ultimate aim of developing medication-based COVID-19 prevention strategies for psychiatric inpatient settings.

\footnotetext{
Catherine L. Clelland (D), PhD, Taub Institute for Research on Alzheimer's Disease and the Aging Brain, Columbia University Irving Medical Center, USA; Department of Pathology and Cell Biology, Columbia University Irving Medical Center, USA; and Department of Psychiatry, Columbia University Irving Medical Center, USA; Krista Ramiah, BS, Clinical Research Department, The Nathan S. Kline Institute for Psychiatric Research, USA: Louisa Steinberg, MD, PhD, Clinical Research Department, Psychiatric Research, USA; Louisa Steinberg, MD, PhD, Clinical Research Depat
The Nathan S. Kline Institute for Psychiatric Research, USA; and Department of The Nathan S. Kline Institute for Psychiatric Research, USA; and Department of
Psychiatry, NYU Grossman School of Medicine, USA; James D. Clelland (D), PhD, Clinical Research Department, The Nathan S. Kline Institute for Psychiatric Research, USA

Correspondence: James Clelland. Email: clelland@nki.rfmh.org

First received 13 Jun 2021, final revision 15 Oct 2021, accepted 21 Oct 2021
}

\section{Supplementary material}

Supplementary material is available online at https://doi.org/10.1192/bjo.2021.1053

\section{Data availability}

The data that support the findings of this study are available from the corresponding author (J.D.C.) upon reasonable request and approval by the Nathan S. Kline Institute institutional review board.

\section{Acknowledgments}

We thank Dr Fabien Tremeau for his useful and informative discussions on this study.

\section{Author contributions}

Concept and design of the study was contributed to by C.L.C. and J.D.C. All authors contributed to the acquisition, analysis and interpretation of data. Statistical analysis was undertaken by C.L.C. Drafting of the initial manuscript was undertaken by C.L.C. and J.D.C. All authors contributed to the critical revision and review of the final manuscript.

\section{Funding}

This research received no specific grant from any funding agency, commercial or not-for-profit sectors.

\section{Declaration of interest}

None.

\section{References}

1 Druss BG. Addressing the COVID-19 pandemic in populations with serious mental illness. JAMA Psychiatry 2020; 77: 891-2.

2 New York State, Office for Mental Health. RPC Statistics Tableau Part 1: Summary Reports. New York State, Office for Mental Health, May 2020 (https://omh.ny.gov/omhweb/tableau/pcs.html).

3 Nemani K, Li C, Olfson M, Blessing EM, et al. Association of psychiatric disorders with mortality among patients with COVID-19. JAMA Psychiatry 2021; 78: 380-6.

4 Muric NN, Arsenijevic NN, Borovcanin MM. Chlorpromazine as a potential antipsychotic choice in COVID-19 treatment. Front Psychiatry 2020; 11: 612347.

5 Hoertel N, Sánchez-Rico M, Vernet R, et al. Observational study of chlorpromazine in hospitalized patients with COVID-19. Clin Drug Investig 2021; 41: 221-33.

6 Hoertel N, Sánchez-Rico M, Vernet R, et al. Observational study of haloperidol in hospitalized patients with COVID-19. PLOS One 2021; 16: e0247122.

7 Hoertel N, Sánchez-Rico M, Vernet R, et al. Association between antidepressant use and reduced risk of intubation or death in hospitalized patients with COVID-19: results from an observational study. Mol Psychiatry [Epub ahead of print] 4 Feb 2021. Available from: https://doi.org/10.1038/s41380-02101021-4.

8 Diez-Quevedo C, Iglesias-González M, Giralt-López M, et al. Mental disorders, psychopharmacological treatments, and mortality in 2150 COVID-19 Spanish inpatients. Acta Psychiatr Scand 2021; 143: 526-34.

9 Lenze EJ, Mattar C, Zorumski CF, et al. Fluvoxamine vs placebo and clinical deterioration in outpatients with symptomatic COVID-19: a randomized clinical trial. JAMA 2020; 324: 2292-300

10 Seftel D, Boulware DR. Prospective cohort of fluvoxamine for early treatment of coronavirus disease 19. Open Forum Infect Dis 2021; 8: ofab050.

11 Mahdavi A M. A brief review of interplay between vitamin $D$ and angiotensinconverting enzyme 2: Implications for a potential treatment for COVID-19. Rev Med Virol 2020; 30: e2119.

$12 \mathrm{Ma} \mathrm{H}$, Zhou T, Heianza Y, Qi L. Habitual use of vitamin D supplements and risk of coronavirus disease 2019 (COVID-19) infection: a prospective study in UK Biobank. Am J Clin Nutr 2021: nqaa381.

13 Oristrell J, Oliva JC, Casado E, et al. Vitamin D supplementation and COVID-19 risk: a population-based, cohort study. I Endocrinol Invest [Epub ahead of print] 17 Jul 2021. Available from: https://doi.org/10.1007/s40618-021-01639-9.

14 Carpinteiro A, Edwards MJ, Hoffmann M, et al. Pharmacological inhibition of acid sphingomyelinase prevents uptake of SARS-CoV-2 by epithelial cells. Cell Rep Med 2020; 1: 100142

15 Carpinteiro A, Gripp B, Hoffmann M, et al. Inhibition of acid sphingomyelinase by ambroxol prevents SARS-CoV-2 entry into epithelial cells. J Biol Chem 2021; 296: 100701 
16 Costa LHA, Santos BM, Branco LGS. Can selective serotonin reuptake inhibitors have a neuroprotective effect during COVID-19? Eur J Pharmacol 2020; 889: 173629.

17 Pashaei Y. Drug repurposing of selective serotonin reuptake inhibitors: could these drugs help fight COVID-19 and save lives? J Clin Neurosci 2021; 88: 163-72.

18 Reynolds HR, Adhikari S, Pulgarin C, et al. Renin-angiotensin-aldosterone system inhibitors and risk of covid-19. N Engl J Med 2020; 382: 2441-8.

19 Hoertel N, Sánchez-Rico M, Gulbins E, et al. Association between FIASMAs and reduced risk of intubation or death in individuals hospitalized for severe COVID-19: an observational multicenter study. Clin Pharmacol Ther [Epub ahead of print] 29 May 2021. Available from: https://doi.org/10.1002/ cpt.2317.

20 Törnquist K, Asghar MY, Srinivasan V, Korhonen L, Lindholm D. Sphingolipids as modulators of SARS-CoV-2 infection. Front Cell Dev Biol 2021; 9: 689854.

21 Hoertel N, Sánchez-Rico M, Cougoule C, Gulbins E, Kornhuber J, Carpinteiro A et al. Repurposing antidepressants inhibiting the sphingomyelinase acid/ceramide system against COVID-19: current evidence and potential mechanisms. Mol Psychiatry [Epub ahead of print] 12 Aug 2021. Available from: https://doi org/10.1038/s41380-021-01254-3.

22 Zimniak $M$, Kirschner $\mathrm{L}$, Hilpert $\mathrm{H}$, et al. The serotonin reuptake inhibitor fluox etine inhibits SARS-CoV-2 in human lung tissue. Sci Rep 2021; 11: 5890.

23 Dechaumes A, Nekoua MP, Belouzard S, Sane F, Engelmann I, Dubuisson J et al. Fluoxetine can inhibit SARS-CoV-2 in vitro. Microorganisms 2021; 9: 339.

24 Schloer S, Brunotte L, Mecate-Zambrano A, Zheng S, Tang J, Ludwig S, et al. Drug synergy of combinatory treatment with remdesivir and the repurposed drugs fluoxetine and itraconazole effectively impairs SARS-CoV-2 infection in vitro. Br J Pharmacol 2021; 178: 2339-2350.

25 Zuo J, Quinn KK, Kye S, Cooper P, Damoiseaux R, Krogstad P. Fluoxetine is a potent inhibitor of coxsackievirus replication. Antimicrob Agents Chemother 2012; 56: 4838-44.
26 Bauer L, Manganaro R, Zonsics B, et al. Fluoxetine inhibits enterovirus replication by targeting the viral $2 \mathrm{C}$ protein in a stereospecific manner. ACS Infect Dis 2019; 5: 1609-23.

27 Johansen LM, DeWald LE, Shoemaker CJ, et al. A screen of approved drugs and molecular probes identifies therapeutics with anti-Ebola virus activity. Sci Transl Med 2015; 7: 290ra89.

28 Greeson JM, Gettes DR, Spitsin S, et al. The selective serotonin reuptake inhibitor citalopram decreases human immunodeficiency virus receptor and coreceptor expression in immune cells. Biol Psychiatry 2016; 80: 33-9.

29 Letendre SL, Marquie-Beck J, Ellis RJ, et al. The role of cohort studies in drug development: clinical evidence of antiviral activity of serotonin reuptake inhibitors and HMG-COA reductase inhibitors in the central nervous system. J Neuroimmune Pharmacol 2007; 2: 120-7.

30 Rosen DA, Seki SM, Fernández-Castañeda A, Beiter RM, Eccles JD, Woodfolk JA, et al. Modulation of the sigma-1 receptor-IRE1 pathway is beneficial in preclinical models of inflammation and sepsis. Sci Transl Med 2019; 11: eaau5266.

31 Hannestad J, DellaGioia N, Bloch M. The effect of antidepressant medication treatment on serum levels of inflammatory cytokines: a meta-analysis. Neuropsychopharmacology 2011; 36: 2452-9.

32 Meltzer DO, Best TJ, Zhang H, Vokes T, Arora V, Solway J. Association of vitamin D status and other clinical characteristics with COVID-19 test results. JAMA Netw Open 2020; 3: e2019722.

33 Clelland JD, Read LL, Drouet V, et al. Vitamin D insufficiency and schizophrenia risk: evaluation of hyperprolinemia as a mediator of association. Schizophr Res 2014; 156: 15-22. 